\title{
Cytochrome P-450 Accumulation and Loss as Controlled by Growth Phase of Saccharomyces cerevisiae: Relationship to Oxygen, Glucose and Ethanol Concentrations
}

\author{
By ANDREW BLATIAK, ${ }^{1}$ DAVID J. KING,${ }^{1} \dagger$ ALAN WISEMAN ${ }^{1 *}$ AND \\ MICHAEL A. WINKLER ${ }^{2}$ \\ ${ }^{1}$ Biochemistry Division, Department of Biochemistry, and ${ }^{2}$ Department of Chemical and Process \\ Engineering, University of Surrey, Guildford, Surrey GU2 $5 X H, U K$
}

(Received 29 April 1986; revised 10 November 1986)

\begin{abstract}
Ethanol induced small amounts of cytochrome P-450 in Saccharomyces cerevisiae NCYC 754 under conditions in which it is not normally detectable. Moreover, in non-growing yeast the existing cytochrome $\mathrm{P}-450$ content was increased by $50 \%$ at a limited range of glucose

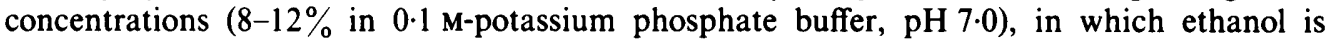
produced by fermentation, possibly at an optimum concentration for induction of cytochrome P-450. Added alkanols, other than ethanol, caused rapid degradation of cytochrome P-450 in nongrowing yeast; the rate of loss was directly related to the lipid solubility of the alkanol. Ethanol therefore favoured the accumulation of cytochrome P-450 in yeast; this may be related to an important putative role of one of the isoenzymes in ethanol-tolerance of the yeast, by the oxidative removal of ethanol from the endoplasmic reticulum of the cell. It is the accumulation of dissolved oxygen, rather than ethanol, that occurs on cessation of yeast growth that is likely to trigger the rapid disappearance of cytochrome P-450 observed at this time.
\end{abstract}

\section{INTRODUCTION}

Cytochrome P-450 enzymes [ $\mathrm{RH}$, reduced-flavoprotein:oxygen oxidoreductase (RHhydroxylating), EC 1.14.14.1] are a group of haemoproteins which catalyse the monooxygenation of both endogenous and exogenous substrates. In Saccharomyces cerevisiae, cytochrome P-450 is involved in the $14 \alpha$-demethylation of lanosterol during the biosynthesis of ergosterol, which is required for yeast membrane synthesis in the endoplasmic reticulum (Aoyama et al., 1984). Cytochrome P-450 from $S$. cerevisiae can also catalyse the monooxygenation of some xenobiotic compounds such as benzo(a)pyrene (Wiseman \& Woods, 1979; King et al., 1984), aminopyrine, p-nitroanisole and caffeine (Sauer et al., 1982), and activate many promutagens to their active mutagenic products (Callen et al., 1980; Kelly \& Parry, 1983).

During growth under strict fermentation conditions, i.e. at glucose concentrations in the range $5-20 \%(\mathrm{w} / \mathrm{v})$, cells of $S$. cerevisiae accumulate large amounts of cytochromes $\mathrm{P}-450$ - more than is apparently required for its endogenous role in the $14 \alpha$-demethylation of lanosterol (Aoyama et al., 1981). The reasons for this accumulation of cytochromes P-450 are not well understood, although factors such as glucose, oxygen and ethanol are believed to be important (Blatiak et al., 1985a; Wiseman et al., 1978; Morita \& Mifuchi, 1984; Del Carratore et al., 1984). Also, under these conditions, glucose repression of mitochondrial proteins, such as cytochrome $a$ $+a_{3}$, occurs, and the yeast therefore derives its energy by fermentation.

$\dagger$ Present address: Department of Protein Biochemistry, Celltech Ltd, 244-250 Bath Road, Slough, Berks SLI 4DY, UK. 
The production of cytochrome P-450 in S. cerevisiae was shown by tetrad analysis to be controlled by a single nuclear gene which was not the structural gene, with at least one modifier gene being involved in the modulation of the amount of cytochrome P-450 produced (King et al., 1983). The identity of these genes is so far unknown. Cyclic AMP may be involved in regulating cytochrome P-450 production in S. cerevisiae by some form of negative control (Wiseman et al., 1978). In addition, cytochrome P-450 may be destroyed in stationary phase culture by a cyclicAMP-dependent covalent modification, as reported for the enzyme from rabbit liver (Muller $e t$ al., 1985), which could be oxygen-concentration dependent. Alternatively, oxygen or oxygenradicals could inactivate the enzyme by directly binding to it.

It has been shown that the production of the yeast haemoproteins iso-1-cytochrome $c$, catalase $\mathrm{T}$ and catalase $\mathrm{A}$ is co-ordinately controlled by glucose, oxygen and haem through the control of mRNA levels (Hortner et al., 1982), although there is also regulation at the post-transcriptional level (Laz et al., 1984). The amount of iso-1-cytochrome $c$ and iso-2-cytochrome $c$ is reduced in yeast grown anaerobically or under glucose repression, whereas the amount of apoprotein of each of cytochrome $b$, cytochrome $c$ peroxidase and cytochrome $c$ is not reduced (Ross \& Schatz, 1976). The production and addition of the haem is therefore the crucial controlling step in these systems, although it should be noted that biosynthesis of the haem moiety occurs in mitochondria (Gudenus et al., 1984). Cytochrome P-450 accumulates in yeast endoplasmic reticulum and is detectable spectrally when yeast is grown semi-anaerobically or under conditions of glucose repression. Different haemoproteins respond differently to glucose, oxygen, haem and ethanol; therefore, studies on the regulation of these systems should contribute to our understanding of the mechanisms involved in the co-ordinate control of the production of eukaryotic proteins.

We have recently shown that cytochrome $\mathrm{P}-450$ is induced in $S$. cerevisiae by small amounts of oxygen, presumably by removal of blocking mechanisms at the genome level, but that at high oxygen concentrations the cytochrome P-450 content is reduced (Blatiak et al., 1985a). In this study we report on the roles of ethanol and glucose in cytochrome P-450 accumulation, and relate this to oxygenation, both during growth and in stationary phase culture.

\section{METHODS}

Organism and growth. S. cerevisiae NCYC 754 was maintained on slopes of Sabouraud dextrose agar. Liquid cultures were grown aerobically at $30^{\circ} \mathrm{C}$ for up to $180 \mathrm{~h}$ in $100 \mathrm{ml}$ medium in a $250 \mathrm{ml}$ conical flask shaken at 120 r.p.m. in an orbital shaker. The medium contained the following (\%,w/v): D-glucose (20); mycological peptone (2); yeast extract (1); and $\mathrm{NaCl}(0 \cdot 5)$.

Yeast cells, harvested in late exponential phase of growth $(35-40 \mathrm{~h})$, were washed and resuspended in $0.1 \mathrm{M}$ potassium phosphate buffer, $\mathrm{pH} 7 \cdot 0$. Members of the $\mathrm{n}$-alkanol series $(6 \%, \mathrm{v} / \mathrm{v})$ were added in separate experiments, and incubated with shaking in a sealed flask (to avoid evaporation of alkanol) at $30^{\circ} \mathrm{C}$ for several hours. Samples were withdrawn at intervals for assay of residual cytochrome P-450.

Assay methods. Cytochrome P-450 was determined directly on whole yeast cells by the reduced carbon monoxide difference spectrum method of Omura \& Sato (1964), using an extinction coefficient of $91 \mathrm{mM}^{-1} \mathrm{~cm}^{-1}$. Ethanol was measured enzymically by using alcohol dehydrogenase and measuring the increase in absorbance at $340 \mathrm{~nm}$ of NADH, using a kit from Boehringer. Glucose was measured by the glucose oxidase method also using a kit from Boehringer.

Batch fermenter growth. Some experiments, as noted later, were done in a 41 microprocessor controlled batch fermenter under optimal conditions for cytochromes $\mathrm{P}-450$ production. These were as follows: $25 \cdot 1{ }^{\circ} \mathrm{C}, \mathrm{pH} 5 \cdot 04$, impeller speed 253 r.p.m., gas flow rate $150 \mathrm{ml} \mathrm{min}^{-1}$, in a medium containing $(\%$, w/v) glucose (14.05), mycological peptone (1.04), yeast extract (1.69) and $\mathrm{NaCl}(0.09)$. The conditions for the growth of yeast in the orbital shaker were also close to the optimum for oxygenation. These were chosen on the basis of previous work (Blatiak et al., 1985a) so that the maximum growth rate of yeast, and the maximum accumulation of cytochrome $\mathrm{P}-450$ in $44 \mathrm{~h}$ could be approached.

\section{RESULTS AND DISCUSSION}

The cytochrome P-450 content of cells of $S$. cerevisiae NCYC 754 was closely linked to the growth phase. It reached a maximum at the end of exponential growth, and fell rapidly soon after the onset of the stationary phase at about $40 \mathrm{~h}$ in medium containing $20 \%(\mathrm{w} / \mathrm{v})$ glucose 


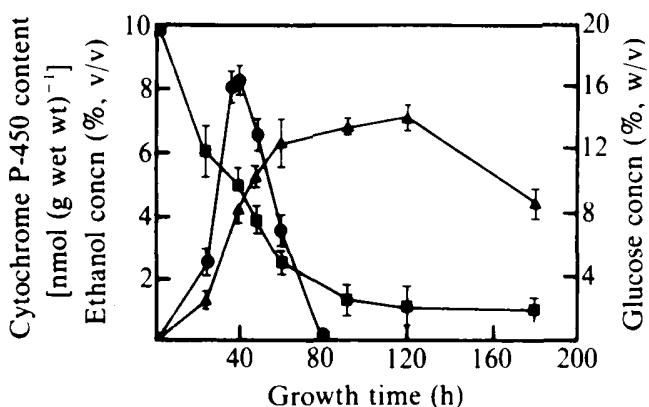

Fig. 1

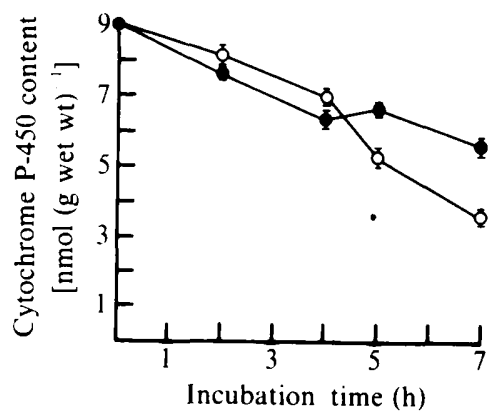

Fig. 2

Fig. 1. Production of cytochrome P-450 during yeast growth in $20 \%(w / v)$ glucose medium correlated with the rise in ethanol concentration and loss of glucose in the growth medium. Cytochrome P-450; D. glucose; $\boldsymbol{\Lambda}$, ethanol. Values are the means of six determinations; error bars indicate SD.

Fig. 2. Effect of ethanol on cytochrome P-450 loss. Yeast cells were resuspended and incubated aerobically with shaking in $100 \mathrm{~mm}$-potassium phosphate buffer, $\mathrm{pH} 7 \cdot 0$, containing no ethanol (O) or $6 \%(\mathrm{v} / \mathrm{v})$ ethanol $(\mathrm{O})$. Values are the means of six determinations; error bars indicate SD.

\section{Table 1. Half-life of cytochrome P-450}

Yeast cells were incubated at $30{ }^{\circ} \mathrm{C}$ in the presence $(6 \%, \mathrm{v} / \mathrm{v})$ of the first five members of the $\mathrm{n}$-alkanol series. Results are the means of two independent determinations (which did not differ significantly).

$\begin{array}{lc}\text { Alkanol } & \begin{array}{c}\text { Half-life } \\ \text { (min) }\end{array} \\ \text { None* } & 420 \\ \text { Methanol } & 130 \\ \text { Ethanol } & 510 \\ \text { Propanol } & 100 \\ \text { Butanol } & 20 \\ \text { Pentanol } & 2.5\end{array}$

* Potassium phosphate buffer only.

(Fig. 1). The half-life of the enzyme was about $20 \mathrm{~h}$ in this residual medium containing about $4 \%$ $(\mathrm{w} / \mathrm{v})$ glucose and $5 \%(\mathrm{v} / \mathrm{v})$ ethanol, as distinct from $7 \mathrm{~h}$ in potassium phosphate buffer (Table 1$)$. As expected, the glucose concentration of the medium declined rapidly with a concurrent increase in ethanol concentration which later fell, due to evaporation losses and the probable use of ethanol as a carbon source under these aerobic, low-glucose conditions. The rapid fall of cytochrome P-450 content in stationary phase has been partially attributed to the decline in the glucose concentration in the growth medium (Wiseman et al., 1975). Another possible factor is the rise in ethanol concentration to approximately $6 \%(\mathrm{v} / \mathrm{v})$ at the end of exponential growth. Our results do not substantiate this view, but imply that loss of cytochrome P-450 is due to buildup of oxygen in the medium after growth ceases, because the yeast is no longer rapidly removing it, resulting in oxygen, or active-oxygen, mediated destruction of the enzyme.

Until recently, it was thought that yeast accumulated ethanol intracellularly to a concentration higher than that in the medium during fermentation (Beaver et al., 1982; Loureiro $\&$ Ferreira, 1983). However, recent work suggests that $S$. cerevisiae does not accumulate ethanol against a concentration gradient but that the yeast plasma membrane is freely permeable to ethanol (Guijarro \& Lagunas, 1984). Ethanol (and other alkanols) are known to have many deleterious effects on yeast at higher concentrations, including an inhibitory effect on growth rate, fermentation rate and viability (Brown et al., 1981; Jones \& Greenfield, 1985). The mechanisms of these effects are unknown, although contributory factors might be the reduction in the uptake of solutes such as glucose (Leao \& Van Uden, 1982 a, b) and amino acids (Leao \& Van Uden, 1984a), passive proton influx across the plasma membrane (Leao \& Van Uden, 
$1984 b$ ) and thermal death (Leao \& Van Uden, 1982). Little is known about the effects of ethanol on the synthesis and degradation of enzymes in stationary phase yeast, although it should not be overlooked that ethanol causes a decrease in water activity and in dielectric constant of the growth medium. The decrease in viability caused by ethanol may be initiated by the interaction of ethanol with lipid components of cell membranes as suggested by Thomas et al. (1978), who demonstrated that yeast cells with added ergosterol were more resistant to ethanol. Casey et al. (1983) showed that supplementation with ergosterol and Tween 80 enabled yeast to grow in much higher concentrations of ethanol. Yeast cytochrome P-450 is a key enzyme for ergosterol biosynthesis (Aoyama et al., 1984), so if ethanol were affecting sterol biosynthesis through an effect on cytochrome P-450, then ergosterol supplementation would indeed be expected to overcome this. However, recent work has suggested that supplementation with sterols including ergosterol could not protect yeast from ethanol inhibition of growth (Walker-Caprioglio et al., 1985). Nevertheless, yeast is particularly sensitive to inhibition of growth and fermentation by ethanol under anaerobic conditions (Aguilera \& Benitez, 1985), presumably when cytochrome $\mathrm{P}-450$ is absent.

The effect of added ethanol on the cytochrome P-450 content of late exponential phase yeast cells was examined. In control incubations in potassium phosphate buffer the cytochrome P-450 content declined in $7 \mathrm{~h}$ to approximately $45 \%$ of that present originally. When ethanol was added little if any change in degradation rate was seen at first, but after $7 \mathrm{~h}$ the cytochrome $\mathrm{P}-450$ content had stabilized to approximately $65 \%$ of that present originally. This may reflect an altered degradation rate after 4-5 h, possibly with additional synthesis of ethanol-induced cytochrome P-450. This effect is also reflected in an increased half-life for cytochrome P-450 when yeast cells were incubated with ethanol compared to the control in buffer alone (Table 1). This effect was not seen with alkanols other than ethanol, all of which accelerated degradation: with increasing chain length of alkanol there was a faster loss of cytochrome P-450 (Table 1). This finding reflects the lipid-solubility of the alkanols: the more lipid-soluble the alkanol, the faster the degradation caused by its addition. The lipid-solubility of alkanols also correlates with many other effects of these compounds on yeast such as on glucose transport and fermentation (Leao \& Van Uden 1985), ammonium transport (Leao \& Van Uden, 1983) and thermal death (Leao \& Van Uden, 1982a,b). This suggests that alkanols interfere with hydrophobic membrane regions, including the endoplasmic reticulum in which cytochrome P-450 is located. Ethanol, however, seems exceptional in its lack of destructive effect on this enzyme.

In yeast grown in medium containing $0.5 \%$ glucose, cytochrome $\mathrm{P}-450$ could not be detected by spectral means (King, 1982); this finding is supported by the work of Aoyama et al. (1981) and Karenlampi et al. (1981). It may be significant that about $35 \%$ more oxygen is dissolved in media containing about $0 \cdot 5-1 \%$ glucose than in the media containing $20 \%$ glucose where P-450 is accumulated. Addition of ethanol to low-glucose media may overcome this effect. To further examine this, cultures of yeast in media containing $0.5 \%$ glucose were supplemented with $1 \%$ ethanol. This resulted in the induction of a small amount of cytochrome P-450 between 20 and $50 \mathrm{~h}$ of incubation (Fig. 3). Ethanol induction of cytochrome P-450 in S. cerevisiae was also observed by Del Carratore et al. (1984) and Morita \& Mifuchi (1984). This induced form of cytochrome P-450 may be similar to that in mammalian liver, in which ethanol induces a form of P-450 which operates via a free-radical mediated mechanism to oxidize ethanol (IngelmanSundberg \& Hagbjork, 1982). The metabolism of 8-methoxypsoralen by $S$. cerevisiae is also induced by ethanol (Prognon et al., 1984), and this may be mediated by a cytochrome P-450 enzyme system. Ethanol is, of course, present in yeast during fermentative growth, under which conditions cytochrome $\mathrm{P}-450$ (cytochrome $\mathrm{P}-448$ isoenzyme) is produced in large quantities. It is likely, therefore, that under these conditions ethanol-induced cytochrome P-450 is synthesized in the endoplasmic reticulum of the yeast cell, and that the main role of this isoenzyme is to oxidize ethanol. Indeed, substrate binding of ethanol to this isoenzyme could stabilize it. The removal of ethanol would be expected to protect the protein biosynthesis system. It should be noted that such forms of cytochrome P-448, functioning by means of free radicals, would be sensitive to inhibition by free radical trapping agents such as the dithiothreitol often used to stabilize cytochromes P-450 during isolation and assay. 


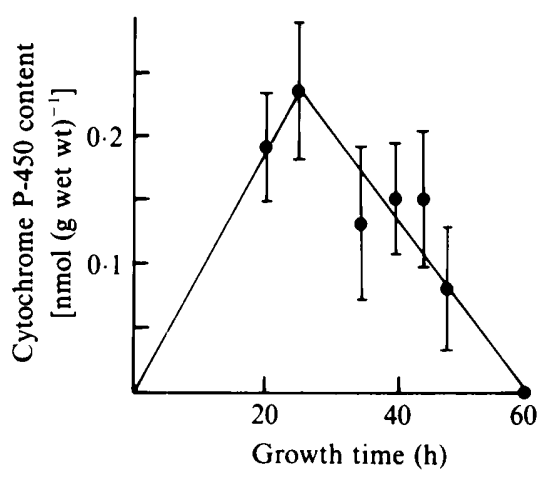

Fig. 3

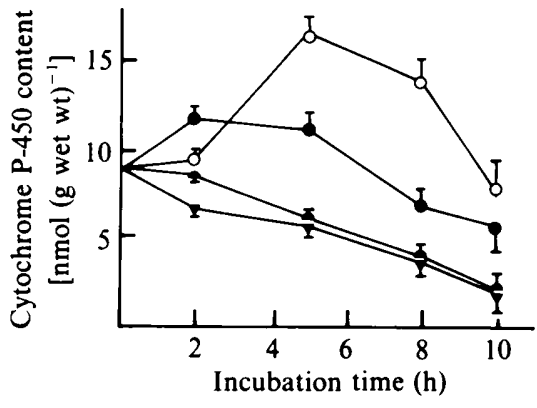

Fig. 4

Fig. 3. Production of cytochrome $P-450$ during yeast growth in $0 \cdot 5 \%(w / v)$ glucose medium containing $1 \%(\mathrm{v} / \mathrm{v})$ ethanol. In the absence of ethanol no cytochrome $\mathrm{P}-450$ could be detected. Values are the means of six determinations; error bars indicate SD.

Fig. 4. Effect of added glucose on the cytochrome P-450 content of yeast incubated aerobically with shaking in $100 \mathrm{~mm}$-potassium phosphate buffer, pH 7.0, containing $0(\nabla), 1(\bullet), 8(O)$ or $12 \%(O)$ $(w / v)$ glucose. Values are the means of six determinations; error bars indicate SD.

High concentrations of glucose in the growth medium cause the accumulation of P-450 in growing yeast. In comparison, the effect of incubating yeast after transfer to non-growth conditions in potassium phosphate buffer, $\mathrm{pH} 7 \cdot 0$, with different concentrations of glucose added, is shown in Fig. 4. With $8 \%(\mathrm{w} / \mathrm{v})$ glucose the already high content of cytochrome P-450 rose after $2-5 \mathrm{~h}$ to $150 \%$ of that present originally, but later fell. With $1 \%(\mathrm{w} / \mathrm{v})$ glucose this effect was not seen, whereas with $5 \%(\mathrm{w} / \mathrm{v})$ glucose some accumulation of P-450 occurred. At higher glucose concentrations less accumulation of P-450 was found, so that with 12 and $20 \%$ $(w / v)$ glucose results were similar. Under these conditions fermentation of the glucose occurs, and it is possible that at $8-12 \%(w / v)$ glucose, fermentation may result in an optimum concentration of ethanol to further induce P-450. No such further accumulation of cytochrome P-450 could be observed using the 41 microprocessor controlled fermenter. However, here the oxygenation may be high enough to destroy the enzyme or, alternatively, to prevent attainment of the optimum concentration of ethanol for induction. In earlier work we found that there was protection from degradation of $\mathrm{P}-450$ by anaerobiosis and other agents that inhibited protein biosynthesis in yeast mitochondria. These would also stop the aerobic metabolism of ethanol (Blatiak et al., 1980).

The large accumulation of $\mathrm{P}-450$ in yeast during rapid fermentative growth on high-glucose media has long been a puzzle when it seems that sufficient P-450 for $14 \alpha$-demethylation of lanosterol can be made even aerobically in low-glucose media (Aoyama et al., 1981). Although growth at a high glucose concentration is very much faster than that at a low glucose concentration, it is doubtful whether all of the extra P-450 produced would be needed for the production of sufficient ergosterol at this faster rate of growth. Therefore, the role of this highglucose induced P-450 is not understood. The induction observed in high-glucose media might be related to the concentration of ethanol produced rather than the glucose concentration. In mammalian systems ethanol is known to induce a specific form of P-450 which has a very high activity towards the oxidation of ethanol to acetaldehyde (Coon et al., 1984), and is also capable of oxidizing a range of other substrates by a free-radical mediated mechanism (IngelmanSundberg \& Hagbjork, 1982). It is likely that in $S$. cerevisiae a comparable system is present, which is also capable of oxidizing some xenobiotics such as benzo(a)pyrene.

It is obvious that a complex mechanism of regulation of P-450 is present in $S$. cerevisiae. Low concentrations of oxygen induce P-450 whereas high concentrations repress or destroy the enzyme (Blatiak et al., 1985a). We have now shown that ethanol, as well as glucose, is also involved in control of P-450 content. It is likely that haem made in mitochondria is also involved, as has been shown for iso-1-cytochrome $c$ (Guarente \& Mason, 1983), so that further 
roles for oxygen are inevitable. The complexity of the regulatory system is perhaps not surprising, as several forms of cytochrome P-450 are now known to exist in $S$. cerevisiae (King $e t$ al., 1982). Part of this work has been reported briefly in a preliminary communication (Blatiak $e t$ al., 1985b).

\section{REFERENCES}

Aguilera, A. \& Benitez, T. (1985). Role of mitochondria in ethanol tolerance of Saccharomyces cerevisiae. Archives of Microbiology 142, 389-392.

Aoyama, Y., Yoshida, Y. \& Sato, R. (1984). Yeast cytochrome P-450 catalysing lanosterol $14 \alpha$-demethylation. II. Lanosterol metabolism by purified $\mathrm{P}-450_{14 \mathrm{DM}}$ and by intact microsomes. Journal of Biological Chemistry 259, 1661-1666.

Aoyama, Y., Okikawa, T. \& Yoshida, Y. (1981). Evidence for the presence of cytochrome $P-450$ functional in lanosterol $14 \alpha$-demethylation in microsomes of aerobically grown respiring yeast. Biochimica et biophysica acta 665, 596-601.

Beaven, M. J., Char pentier, C. \& Rose, A. H. (1982). Production and tolerance of ethanol in relation to phospholipid fatty-acyl composition in Saccharomyces cerevisiae NCYC 431. Journal of General Microbiology 128, 1447-1455.

Blatiak, A., Gondal, J. A. \& Wiseman, A. (1980). Mechanism of degradation of cytochrome P-450 in non-growing Saccharomyces cerevisiae: anaerobic, chloramphenicol and cycloheximide as protective agents. Biochemical Society Transactions 8, 711-712.

Blatiak, A., King, D. J., Wiseman, A., Salihon, J. \& WINKLER, M. A. (1985a). Enzyme induction by oxygen in the accumulation of cytochrome P-450 during batch fermentations in $20 \%$ D-glucose with Saccharomyces cerevisiae. Enzyme and Microbial Technology 7, 553-556.

Blatiak, A., King, D. J. \& Wiseman, A. (1985b). Effect of alcohols and of glucose on the level of cytochrome P-450 in Saccharomyces cerevisiae after resuspension in buffer. Biochemical Society Transactions 13, 924.

Brown, S. W., Oliver, S. G., Harrison, D. E. F. \& Righelato, R. C. (1981). Ethanol inhibition of yeast growth and fermentation: differences in the magnitude and complexity of the effect. European Journal of Applied Microbiology and Biotechnology 11, 151155.

Callen, D. F., Wolf, C. R. \& Philpot, R. M. (1980). Cytochrome P-450 mediated genetic activity and cytotoxocity of seven halogenated aliphatic hydrocarbons in Saccharomyces cerevisiae. Mutation Research 77, 55-63.

Casey, G. P., Magnus, C. A. \& Ingledew, W. M. (1983). High gravity brewing: nutrient enhanced production of high concentrations of ethanol by brewing yeast. Biotechnology Letters 5, 429-434.

CoOn, M. J., Koop, D. R., ReEve, L. E. \& CRUmp, B. L. (1984). Alcohol metabolism and toxicity: role of cytochrome P-450. Fundamental Applied Toxicology 4, 134-143.

Del Carratore, R., Morganti, C., Galli, A. \& Bronzetta, G. (1984). Cytochrome P-450 inducibility by ethanol and 7-ethoxycoumarin $O$-deethylation in $S$. cerevisiae. Biochemical and Biophysical Research Communications 123, 186-193.
Guarente, L. \& Mason, T. (1983). Heme regulates transcription of the $\mathrm{CYCl}$ gene of $S$. cerevisiae via an upstream activation site. Cell 32, 1279-1286.

Gudenus, R., Spence, A., Hartig, A., Smith, M. \& RuIS, H. (1984). Regulation of transcription of the Saccharomyces cerevisiae $\mathrm{CYCl}$ gene: identification of a DNA region involved in heme control. Current Genetics 8, 45-48.

GuiJarRo, J. M. \& LAGUNAS, R. (1984). Saccharomyces cerevisiae does not accumulate ethnol against a concentration gradient. Journal of Bacteriology 160, 874-878.

Hortner, H., Ammerer, G., Hartter, E., Hamilton, B., RytKA, J., Bilinski, T. \& RUIS, H. (1982). Regulation of synthesis of catalases and iso-1cytochrome $c$ in Saccharomyces cerevisiae by glucose, oxygen and heme. European Journal of Biochemistry 128, 179-184.

INGELMAN-SUNDBURY, M. \& HAGBJORK, A. L. (1982). On the significance of the cytochrome P-450dependent hydroxyl radical mediated oxygenation mechanism. Xenobiotica 12, 673-686.

Jones, R. P. \& Greenfield, P. F. (1985). Replicative inactivation and metabolic inhibition in yeast ethanol fermentations. Biotechnology Letters 7, 223228.

Karenlampi, S. O., Martin, E. \& Hanninen, O. O. P. (1981). Effect of carbon source on the accumulation of cytochrome P-450 in the yeast Saccharomyces cerevisiae. Biochemical Journal 194, 407-413.

Kelly, D. \& ParRY, J. M. (1983). Metabolic activation of cytochrome P-450/P-448 in the Saccharomyces cerevisiae. Mutation Research 108, 147-159.

KING, D. J. (1982). Studies on the biosynthesis and specificity of cytochrome P-450 in the yeast Saccharomyces cerevisiae. PhD thesis, University of Surrey, UK

KING, D. J., AzARI, M. R. \& Wiseman, A. (1982). The induction of cytochrome P-448 dependent benzo(a) pyrene hydroxylase in Saccharomyces cerevisiae. Biochemical and Biophysical Research Communications 105, 1115-1121.

King, D. J., Wiseman, A. \& Wilkie, D. (1983). Studies on the genetic regulation of cytochrome P-450 production in Saccharomyces cerevisiae. Molecular and General Genetics 192, 466-470.

King, D. J., AZARI, M. R. \& Wiseman, A. (1984). Studies on the properties of highly purified cytochrome P-448 and its dependent activity benzo(a)pyrene hydroxylase from Saccharomyces cerevisiae. Xenobiotica 14, 187-206

Laz, T. M., Pietras, D. F. \& Sherman, F. (1984). Differential regulation of the duplicated isocytochrome $c$ genes in yeast. Proceedings of the National Academy of Sciences of the United States of America 81, 4475-4479.

LEAO, C. \& VAN UDEN, N. (1982a). Effects of ethanol and other alkanols on the kinetics and the activation 
parameters of thermal death in Saccharomyces cerevisiae. Biotechnology and Bioengineering 24, 26012604.

LEAO, C. \& VAN UdEN, N. (1982b). Effects of ethanol and other alkanols on the glucose transport system of Saccharomyces cererisiae. Biotechnology and Bioengineering 24, 2601-2604.

LEAO, C. \& VAN UDEN, N. (1983). Effects of ethanol and other alkanols on the ammonium transport system of Saccharomyces ceretisiae. Biotechnology and Bioengineering 25, 2085-2090.

LEAO, C. \& VAN UDEN, N. (1984a). Effects of ethanol and other alkanols on the general amino acid permease of Saccharomyces cerevisiae. Biotechnology and Bioengineering 26, 403-405.

LEAO, C. \& VAN UDEN, N. (1984b). Effects of ethanol and other alkanols on passive proton influx in the yeast Saccharomyces cerevisiae. Biochimica et biophysica acta 774, 43-48.

LeaO, C. \& VAN UdEN, N. (1985). Effects of ethanol and other alkanols on the temperature relations of glucose transport and fermentation in Saccharomyces cerevisiae. Applied Microbiology and Biotechnology 22, 359-363.

Loureiro, V. \& Ferreira, H. G. (1983). On the intracellular accumulation of ethanol in yeast. Biotechnology and Bioengineering 25, 2263-2269.

Morita, T. \& MifuChI, I. (1984). Ethanol enhancement of cytochrome P-450 in yeast Saccharomyces cerevisiae D7. Chemical Pharmacology Bulletin 32, 1624-1627.

Muller, R., Schmidt, W. E. \& Stier, A. (1985). The site of cyclic AMP-dependent protein kinase catalysed phosphorylation of cytochrome P-450 LM2. FEBS Letters 187, 21-24.

ONURA, T. \& SaTo, R. (1964). The carbon monoxide binding pigment of liver microsomes. I. Evidence for its haemoprotein in nature. Journal of Biological Chemistry 239, 2370-2379.
Prognon, P., Blais, J., Vigny, P., Averbeck, D., AverbecK , S. \& Gond, A. (1984). The metabolism of 8-methoxypsoralen by Saccharomyces cerevisiae. Evidence for an inducing effect of ethanol. Il Farmaco Edizione Scientifica 39, 739-751.

Ross, E. \& Schatz, G. (1976). Cytochrome $c_{1}$ of Baker's yeast. II. Synthesis on cytoplasmic ribosomes and influences of oxygen and heme on accumulation of the apoprotein. Journal of Biological Chemistry 251, 1997-2004.

Sauer, M., Kappeli, O. \& Fiechter, A. (1982). Comparison of the cytochrome $\mathrm{P}-450$ containing monooxygenases originating from two different yeasts. In Cytochrome P-450: Biochemistry, Biophysics and Entironmental Implications, pp. 453-457. Edited by E. Hietanen, M. Laitinen \& O. Hanninen. Amsterdam: Elsevier Biomedical Press.

Thomas, D. S., Hossack, J. A. \& Rose, A. H. (1978). Plasma membrane lipid composition and ethanol tolerance in Saccharomyces cerevisiae. Archives of Microbiology 117, 239-245.

Walker-Caprioglio, H. M., Rodrigues, R. J. \& PARKS, L. W. (1985). Recovery of Saccharomyces cerevisiae from ethanol-induced growth inhibition. Applied and Entironmental Microbiology 50, 685-689.

WiSEMAN, A. \& WoODS, L. F. J. (1979). Benzo(a)pyrene metabolites formed by the action of yeast cytochrome P-450/P-448. Journal of Chemical Technology and Biotechnology 29, 320-324.

Wiseman, A., Lim, T. K. \& MCCloud, C. (1975). Relationship of cytochrome P-450 to growth phase of Brewer's yeast in $1 \%$ or $20 \%$ glucose medium. Biochemical Society Transactions 3, 276-278.

Wiseman, A., Lim, T. K. \& Woods, L. F. J. (1978). Regulation of the biosynthesis of cytochrome P-450 in Brewer's yeast. Role of cyclic AMP. Biochimica et biophy'sica acta 544, 615-623. 